\title{
Epidemiology of COVID-19 in Northern Ireland, 26 February 2020-26 April 2020
}

\section{Original Paper}

Cite this article: Pett $J$, McAleavey $P$, McGurnaghan P, Spiers R, O'Doherty M, Patterson L, Johnston J (2021). Epidemiology of COVID-19 in Northern Ireland, 26 February 2020-26 April 2020. Epidemiology and Infection 149, e36, 1-10. https://doi.org/10.1017/ S0950268821000224

Received: 12 August 2020

Revised: 22 January 2021

Accepted: 27 January 2021

\section{Key words:}

Coronavirus; COVID-19; pandemic;

surveillance; transmission

Author for correspondence:

J. Pett, E-mail: joseph.pett@phe.gov.uk (c) The Author(s), 2021. Published by Cambridge University Press. This is an Open Access article, distributed under the terms of the Creative Commons Attribution licence (http://creativecommons.org/licenses/by/4.0/), which permits unrestricted re-use, distribution, and reproduction in any medium, provided the original work is properly cited.

\section{J. Pett ${ }^{1,2}$ (D, P. McAleavey ${ }^{1}$, P. McGurnaghan ${ }^{1}$, R. Spiers ${ }^{1}$, M. O'Doherty ${ }^{1}$, L. Patterson ${ }^{1}$ (D) and J. Johnston ${ }^{1}$}

${ }^{1}$ Public Health Agency Northern Ireland, Belfast, Northern Ireland and ${ }^{2}$ UK Field Epidemiology Training Programme, Global Public Health Division, Public Health England, London, UK

\section{Abstract}

This paper describes the epidemiology of coronavirus disease 2019 (COVID-19) in Northern Ireland (NI) between 26 February 2020 and 26 April 2020, and analyses enhanced surveillance and contact tracing data collected between 26 February 2020 and 13 March 2020 to estimate secondary attack rates (SAR) and relative risk of infection among different categories of contacts of individuals with laboratory confirmed severe acute respiratory syndrome-coronavirus-2 (SARS-CoV-2) infection. Our results show that during the study period COVID-19 cumulative incidence and mortality was lower in NI than the rest of the UK. Incidence and mortality were also lower than in the Republic of Ireland (ROI), although these observed differences are difficult to interpret given considerable differences in testing and surveillance between the two nations. SAR among household contacts was $15.9 \%$ (95\% CI 6.6\%-30.1\%), over 6 times higher than the SAR among 'high-risk' contacts at 2.5\% (95\% CI $0.9 \%-5.4 \%$ ). The results from logistic regression analysis of testing data on contacts of laboratory-confirmed cases show that household contacts had 11.0 times higher odds (aOR: 11.0, 95\% CI 1.7-70.03, $P$-value: 0.011$)$ of testing positive for SARS-CoV-2 compared to other categories of contacts. These results demonstrate the importance of the household as a locus of SARS-CoV-2 transmission, and the urgency of identifying effective interventions to reduce household transmission.

\section{Introduction}

On 31 December 2019, a cluster of cases of pneumonia of unknown origin was reported to the World Health Organisation (WHO) by Chinese authorities in Wuhan [1]. These are now known to have been cases of coronavirus disease 2019 (COVID-19), caused by the virus severe acute respiratory syndrome-coronavirus-2 (SARS-CoV-2), the early epidemiology and characteristics of which are described extensively elsewhere [2-10].

The first suspected COVID-19 case in Northern Ireland (NI) was reported to the Public Health Agency (PHA) and tested on 22 January 2020. The first laboratory-confirmed case reported in the UK occurred in England on 29 January 2020. The first laboratory-confirmed case in NI was reported on 26 February 2020, and on 29 February 2020, COVID-19 was added to the schedule of notifiable diseases in NI [11].

This paper describes the epidemiology of COVID-19 in NI between 26 February 2020 and 26 April 2020. It also describes enhanced surveillance data on the first 39 cases reported in NI, for whom enhanced surveillance data were available and contact tracing and monitoring was carried out.

\section{Methods}

\section{Case definitions}

The UK has used five different case definitions for COVID-19 since the pandemic began, and these are presented in Appendix 1.

\section{Contact definitions}

Contacts of cases are categorised as household, high and low risk (Appendix 2). During the study period, contacts were only offered testing if they reported symptoms included in the case definition in use at the time.

\section{SARS-CoV-2 laboratory testing}

Testing was made available from PHE's National Reference Laboratory for all of the UK on 24 January 2020. On 7 February 2020, SARS-CoV-2 the Regional Virus Laboratory, Belfast Health 
and Social Care (HSC) Trust began conducting testing locally. Testing was rolled out to the other four HSC Trust labs between 23 March 2020 and 13 May 2020.

Between 22 January 2020 and 13 March 2020, testing was performed for all individuals meeting the case definition in use at the time testing was carried out. On 13 March 2020, testing was expanded to HSC workers and members of their household who meet the suspected case definition but do not require hospitalisation. Testing was expanded again on 4 April 2020 to include other 'key workers' (see Appendix 3 for list of key worker categories) who meet the suspected case definition but do not require hospitalisation.

\section{Data sources}

\section{Routine virology data}

The PHA Health Protection Directorate laboratory surveillance system collates SARS-CoV-2 laboratory data on all tests from HSC Trust laboratories. Laboratory data are then collated to enable monitoring of individuals rather than tests performed by laboratories using the Organism-Patient-Illness-Episode (OPIE) principle [12].

Data on all individuals with a positive test result between 26 February 2020 and 26 April 2020 were extracted from the PHA Health Protection laboratory surveillance system. These data also include date of birth, sex and residential postcode for each case.

\section{Enhanced surveillance data}

The PHA Health Protection surveillance team developed a bespoke electronic-enhanced surveillance system using web-based forms for collecting surveillance information on cases and contacts, aligned with PHE protocol adapted from WHO guidance [13]. Contacts were identified via phone calls with the index case, or a contact of the index case where the index case was unable to provide these data.

Enhanced surveillance and contacts data for the 39 cases reported in NI during the initial period of contact tracing were extracted from this dataset, including demographics, travel history, exposures, occupation, symptoms and outcomes (see Appendix 4). Contact data include demographics and categorisation into household, high risk or low risk (Appendix 2). During the initial phone call with contacts of index cases, contacts were asked if they were symptomatic and if they reported symptoms testing was arranged. Where contacts reported no symptoms, they were advised to contact PHA to arrange testing if they experienced symptoms over the 14 days since their last contact with the index case. Where data were missing from the enhanced surveillance datasets, individual case records from HPZone, an electronic case-management system in use throughout the UK, were reviewed to complete missing fields wherever possible.

\section{Data analysis}

We described COVID-19 cases from the routine virology surveillance dataset by age, sex, local government district (LGD), and deprivation quintile. We also described COVID-19 cases from the enhanced case and contact surveillance datasets by age, sex, exposures, symptoms and outcome. We estimated secondary attack rates (SAR) and secondary clinical attack rates in household, high-risk and low-risk contacts and by age group. We then estimated incubation periods and serial intervals using symptom onset dates in transmission pairs where data were available. Where the secondary case's reported date of symptom onset was the same as the date of most recent contact with the primary case, one day was assigned as the minimum incubation period.

We then carried out multivariate logistic regression analysis to estimate the association between contact risk category, age and sex and testing positive for SARS-CoV-2. We calculated crude and adjusted odds ratios, and 95\% credible intervals (CI).

\section{Results}

\section{Descriptive epidemiology in NI}

In all, 3380 individuals tested positive for SARS-CoV-2 in NI during the study period (Fig. 1) giving NI a cumulative incidence per 100000 population of 179.6 (95\% CI $173.6-185.8$ ). This is lower than cumulative incidence in England at 208.4 (95\% CI 207.2209.6) per 100000 population [14], and below Wales at 308.7 (95\% CI 302.6-314.9) [15], and Scotland at 189.3 (95\% CI 185.7-193.0) [16] over this period.

This is also lower than the Republic of Ireland (ROI), where cumulative incidence was 392.8 (95\% CI 387.3-398.4) per 100 000 population [17]. This is likely explained by differences in testing and contact tracing policy between NI and the ROI, and these different approaches to surveillance and policy make interpretation of total case numbers difficult to interpret.

\section{Crude COVID-19 mortality rate}

In total, 334 individuals with laboratory-confirmed SARS-CoV-2 infection died in NI over the study period, giving a crude COVID-19 mortality rate of 17.7 per 100000 population (95\% CI 15.9-19.8). This is lower than the crude COVID-19 mortality rates per 100000 population in England, Scotland and Wales, which are 38.9 (95\% CI 38.3-39.4), 22.9 (95\% CI 21.6-24.2) and 28.6 (95\% CI 26.8-30.5), respectively. It is also lower than the crude mortality rate in the ROI of 20.6 per 100000 population (95\% CI 19.3-21.9), although the 95\% CIs for NI and the ROI overlap slightly.

\section{Age and sex distribution}

Cases ranged in age from $<1$ month to 104 years old (median: 57.1, IQR: 40.5, 80.3). In all, 61.9\% (2093) were aged 50 or over and $59.8 \%$ (2022) were female individuals (Table 1). The age and sex profile of reported cases in NI has changed over time (Figs 2 and 3), with the number of cases that are female and in older age groups increasing relative to males and younger age groups. Cases were reasonably evenly distributed between deprivation quintiles.

\section{Descriptive epidemiology of enhanced surveillance and contact tracing data}

Between 26 February 2020 and 7 March 2020, all cases [10] were associated with travel to an affected area that was included in the UK case definition (Fig. 4). Seven out of the eight cases between 7 March 2020 and 9 March 2020 were part of two clusters linked to one primary case who tested positive on 6 March 2020.

On 9 March 2020 the first sporadic case (a case without a relevant travel history or known epidemiological link to a case or cluster in NI or elsewhere in the UK occurred) tested positive. 


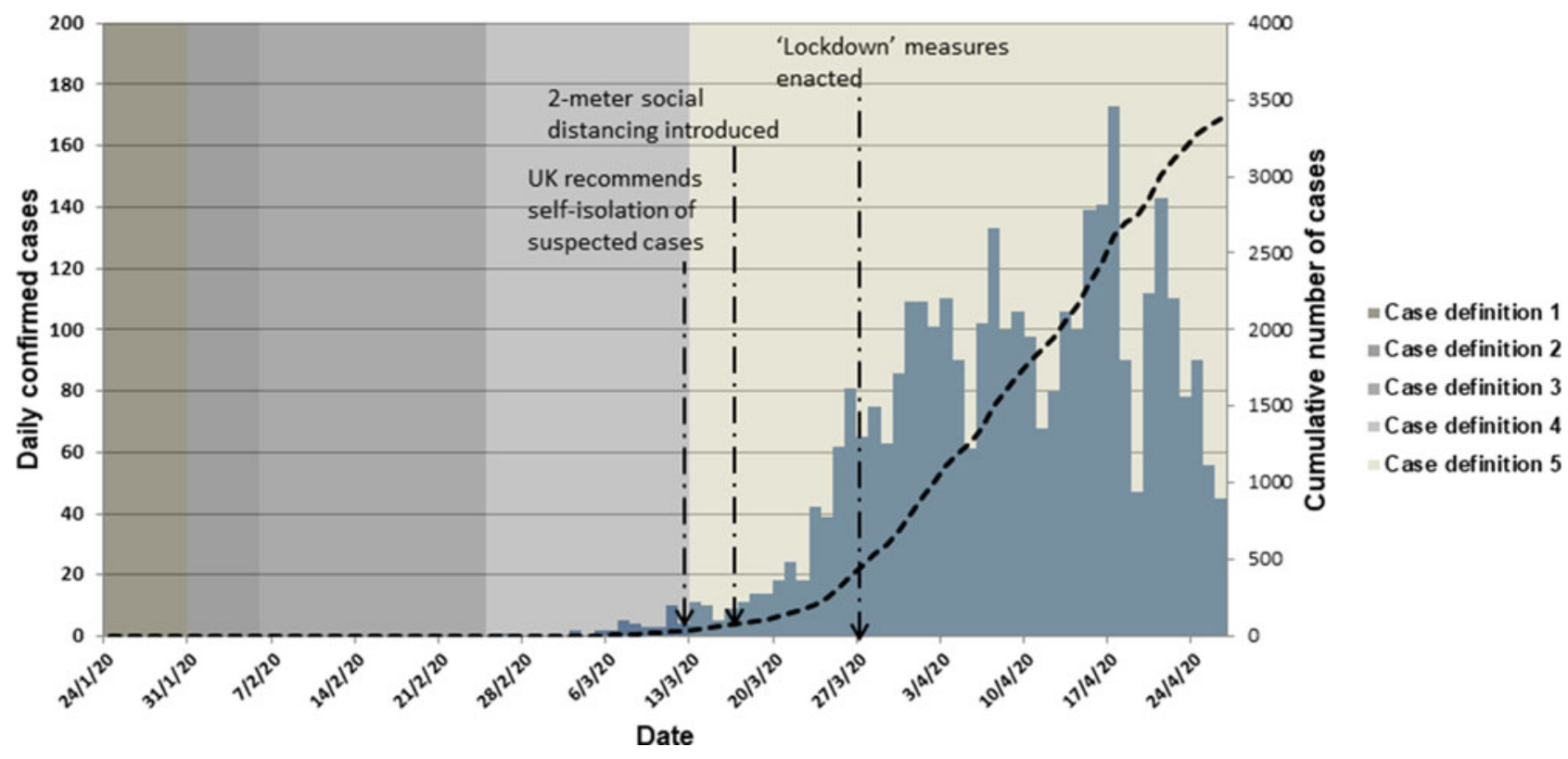

Fig. 1. Daily number of confirmed COVID-19 cases in NI, by reporting date, 24 January 2020 to 26 April 2020 ( $n=3380$ ).

A further four sporadic cases occurred between 11 March 2020 and 15 March 2020.

\section{Exposures}

Between 26 February 2020 and 13 March 2020, 51.3\% [18] of all cases travelled to a known affected area outside the UK in the 14 days before notification, of whom $10(50.0 \%)$, seven $(35.0 \%)$ and two $(10.0 \%)$ travelled to Italy, Austria and France, respectively. One $(5.0 \%)$ individual had recently returned from a cruise. In all, 48.7\% [19] reported contact with an individual or individuals with COVID-19-like symptoms in the previous 14 days, and $33.3 \%$ [13] were contacts of known cases in NI (Fig. 5). A percentage of 7.7 [3] were employed as healthcare workers (HCW) at the time of reporting, two of whom were primary cases and one of whom was a secondary case (Table 2).

\section{Age-sex distribution}

Cases identified through enhanced surveillance ranged in age from 13 to 86 years old, with the majority (64.1\%) aged below 50 years (Table 3); 59.0\% [20] of the cases were male. The age and sex distribution of these early cases differs from those reported after 13 March 2020, and this is likely due to changes to the UK case definition and testing policy which meant the majority of cases reported to PHA were hospitalised patients, individuals living in care homes, and to a lesser extent, healthcare and other key workers.

\section{Symptoms and co-morbidities}

Data on reported symptoms and co-morbidities were available for $36(92.3 \%)$ cases. Of these, $91.7 \%$ [21] reported at least one symptom at the time of, or in the 7 days prior to, notification. Of these, $69.4 \%$ [22] reported fever, $66.6 \%$ [23] reported a cough and $22.2 \%$ [8] reported shortness of breath. The least commonly reported symptoms were delirium (2.9\%), myalgia (2.9\%) and fatigue (2.9\%). Three (8.3\%) cases reported experiencing no symptoms (Table 4).

\section{Other outcomes}

Data on hospital admission and mortality during the period 26 February 2020 to 13 March 2020 were available for 35 (89.7\%) and $39(100.0 \%)$ cases, respectively. Of these, $31.4 \%$ [11] were admitted to hospital, and one case (2.9\%) died.

\section{Analytical epidemiology of enhanced surveillance and contact tracing data}

Complete information on contacts of cases was available for 27 cases (69.2\%). Ten (25.6\%) cases were identified after the suspension of contact tracing on 13 March 2020, and so contact tracing data were not available for these individuals. Five $(50.0 \%)$ of these had a positive test result on or after 13 March 2020, and five (50.0\%) were contacts of confirmed cases and had a positive test result within 7 days of 13 March 200, which were able to identify through linking contact tracing data to virology data. The specimen date for the last of these cases was 18 March 2020. For the two $(5.1 \%)$ remaining cases for whom no contact tracing data were available, we were unable to determine whether this was because they reported having no household, high- or low-risk contacts, or if these data were simply not collected.

Totally, 392 contacts were identified from the 27 cases for whom contact tracing data were available, with the median number of contacts per case was 4 (min: 1.0, max: 57.0, IQR: 2.5, 17.5). Overall, 44 (11.5\%), 238 (60.7\%) and $110(28.1 \%)$ were household, high- and low-risk contacts, respectively. Of the 392 contacts, 44 (11.2\%) were tested for SARS-CoV-2, of which 13 (29.5\%) were positive, one (2.3\%) initially tested negative before testing positive 12 days later and the remaining $30(68.2 \%)$ were negative.

\section{Transmission dynamics}

Between 26 February 2020 and 18 March 2020, 13 (33.3\%) cases were contacts of previously reported cases within NI. Seven (53.8\%) were household contacts, six (46.2\%) were high-risk contacts and there were no cases reported among low-risk contacts. 
Table 1. Demographic characteristics of confirmed COVID-19 cases in NI, 26 February 2020 to 26 April 2020, $n=3380$

\begin{tabular}{|c|c|c|}
\hline Demographics & $n$ & $\%$ \\
\hline Female & 2022 & 59.8 \\
\hline Age range (years) & $0-104$ & \\
\hline Median age & 57.1 & \\
\hline Age group & $n$ & $\%$ \\
\hline$<5$ & 8 & 0.2 \\
\hline $5-10$ & 11 & 0.3 \\
\hline $10-20$ & 54 & 1.6 \\
\hline $20-30$ & 372 & 11.0 \\
\hline $30-40$ & 376 & 11.1 \\
\hline $40-50$ & 466 & 13.8 \\
\hline $50-60$ & 540 & 16.0 \\
\hline $60-80$ & 697 & 20.6 \\
\hline $80+$ & 856 & 25.3 \\
\hline LGD & $n$ & $\%$ \\
\hline Belfast & 1058 & 31.3 \\
\hline Armagh, Banbridge and Craigavon & 398 & 11.8 \\
\hline Lisburn and Castlereagh & 341 & 10.1 \\
\hline Antrim and Newtonabbey & 306 & 9.1 \\
\hline Mid and East Antrim & 234 & 6.9 \\
\hline Ards and North Down & 210 & 6.2 \\
\hline Newry, Mourne and Down & 201 & 5.9 \\
\hline Causeway Coast and Glens & 185 & 5.5 \\
\hline Derry and Strabane & 172 & 5.1 \\
\hline Mid Ulster & 166 & 4.9 \\
\hline Fermanagh and Omagh & 68 & 2.0 \\
\hline Residence outside NI or unknown & 41 & 1.2 \\
\hline Deprivation quintile & $n$ & $\%$ \\
\hline 1 (least deprived) & 701 & 20.7 \\
\hline 2 & 601 & 17.8 \\
\hline 3 & 747 & 22.1 \\
\hline 4 & 723 & 21.4 \\
\hline 5 (most deprived) & 825 & 24.4 \\
\hline Unknown & 41 & 1.2 \\
\hline
\end{tabular}

SAR and clinical attack rates for different categories of contact and by age group are shown in Table 5 . We estimate that the SAR among household contacts $(15.9 \%, 95 \%$ CI $6.6 \%-30.1 \%)$ is over 6 times higher than among high-risk contacts $(2.5 \%$, 95\% CI $0.9 \%-$ 5.4\%). Six (46.2\%) secondary cases' earliest reported contact with the primary case was before the primary case's reported date of symptom onset, of which two secondary cases' (33.3\%) only reported contact with the primary case was before the primary case's reported onset of symptoms, indicating possible presymptomatic transmission.

Age data were available for 259 (65.7\%) contacts. By age group, the highest attack rate observed was in $20-30$-year olds at $19.3 \%$.
There were no cases reported in under 5-year, 5-10-year olds, 6070 -year olds and contacts aged 80 and older. The clinical attack rate was highest in 70-80-year olds at $10.0 \%$ (95\% CI $0.3-44.5 \%$ ). The $95 \%$ CIs for all age groups overlap, however, and given the small number of secondary cases in our study we were unlikely to detect evidence of variation in attack rates by age group.

Six clusters produced the 13 secondary cases identified in NI. The number of secondary cases in these clusters ranged from one to four, with a median cluster size of two. The median minimum incubation period in the six clusters was 2 days (range: 1-12) (Table 6), the median maximum incubation period was 6 (range: 3-16), and the median serial interval was 6 (range: 2-13).

To further investigate infection risk among different categories of contacts, we performed a multivariate logistic regression comparing test positivity in household contacts of confirmed cases who were tested for SARS-CoV-2 compared to high- and low-risk contacts. Analysis of data on the 44 contacts tested shows that, after adjusting for age and sex, household contacts had 11.0 (95\% CI 1.7-70.3, $P=0.011$ ) times higher odds of testing positive for SARS-CoV-2 compared to high- and low-risk contacts (Table 7). We found no evidence of association between age (OR: $1.0,95 \%$ CI $0.9-1.1, P=0.884$ ) or sex (OR: $0.5,95 \% \mathrm{CI}$ $0.1-2.9, P=0.462)$ and testing positive for SARS-CoV-2.

\section{Limitations}

This paper has at least six limitations.

First, the enhanced surveillance and contact tracing data cover the early stages of the COVID-19 epidemic in NI, and largely represents the epidemiology of imported cases $(53.9 \%$ of cases acquired their infection outside of NI) and their contacts. It is therefore potentially less representative of the period of sustained community transmission that followed introduction of SARS-CoV-2 in NI [24].

Second, as identification of contacts was dependent on selfreporting by index cases, household contacts are likely overrepresented in our sample which may bias our results.

Third, cases reported after the suspension of contact tracing on 13 March 2020 represent the epidemiology of hospitalised cases only, and to a lesser extent, healthcare and other key workers that were offered testing. This limits the generalisability of the findings presented here.

Fourth, throughout the study period, testing was only offered to individuals who met the epidemiological criteria, were symptomatic, and whose symptoms were among those specified in the case definition in use at the time their symptoms presented. This means symptomatic cases may not have been offered testing because their symptoms were not in the case definition. Given UK testing policy, asymptomatic individuals ${ }^{1}$ were also unlikely to be tested and therefore asymptomatic cases were less likely to be detected, although three cases in the enhanced surveillance and contact tracing dataset were tested despite not reporting any symptoms.

The potential impact of this is two-fold. Misclassification of cases means total case numbers are underestimated and biased towards identifying cases with more severe disease, and estimates of attack rates and the risk of infection in different categories of contacts will also be biased, although in the latter case this non-

\footnotetext{
${ }^{1}$ Testing may have been arranged at the discretion of their primary care provider, or the individuals may have reported symptoms to their primary care provider which they did not later report to PHA surveillance staff.
} 


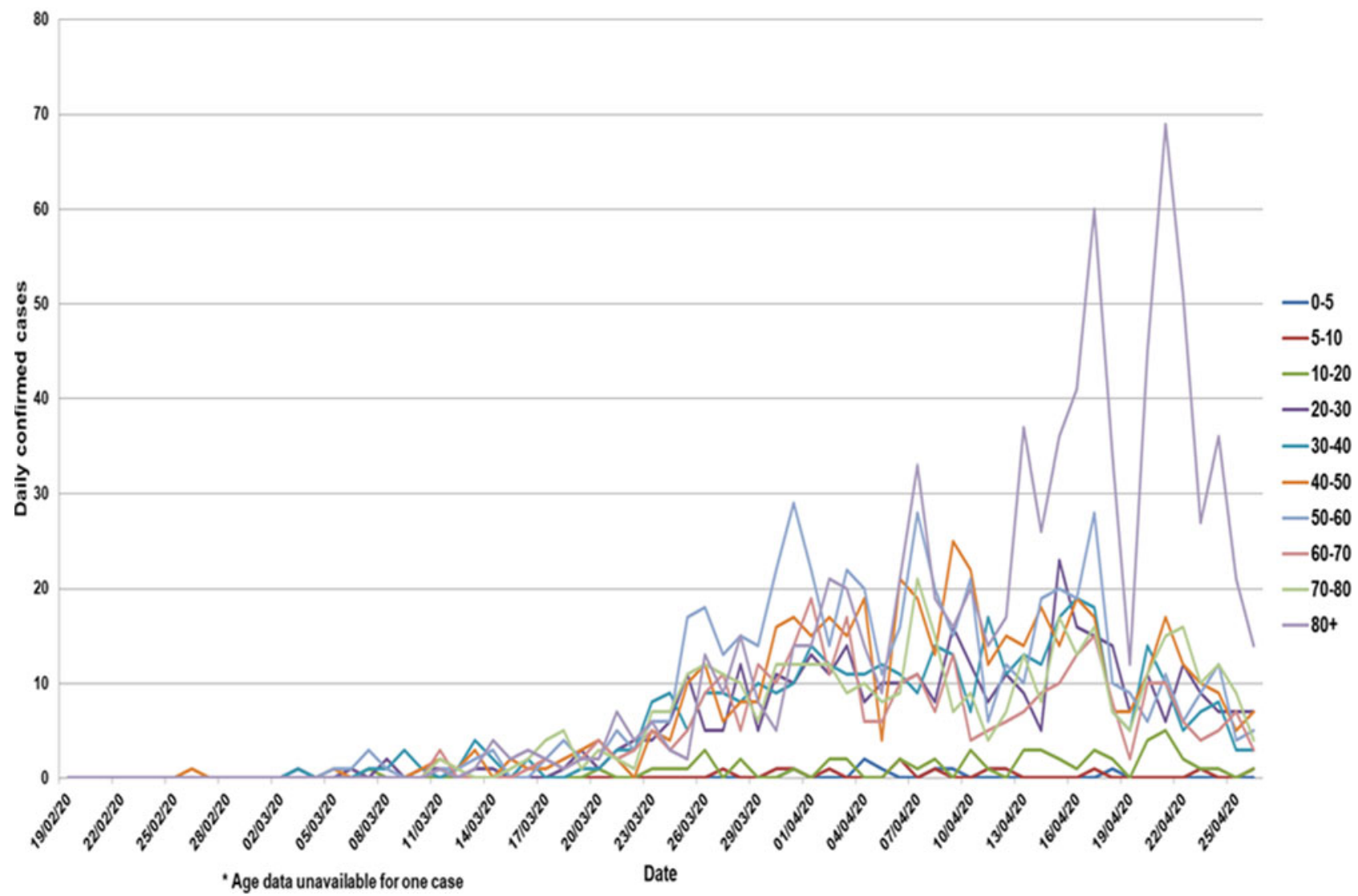

Fig. 2. Daily COVID-19 cases by age group and specimen date, NI, 19 February 2020 to 26 April $2020(n=3379)^{\star}$.

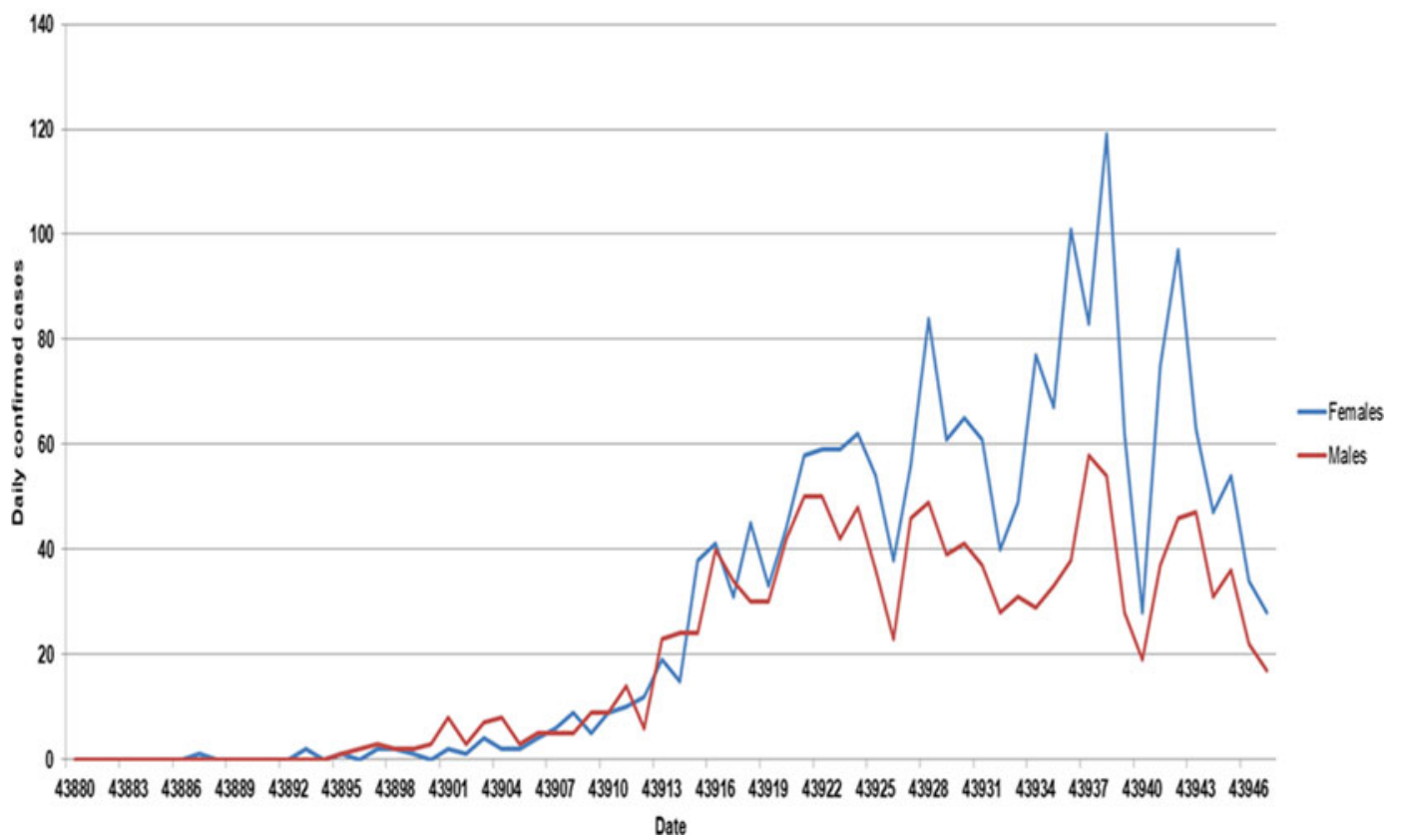

Fig. 3. Daily COVID-19 cases by sex and specimen date, NI, 19 February 2020 to 26 April $2020(n=3380)$.

differential misclassification of SARS-CoV-2 positivity may be less likely to affect the estimate of the relative risk of infection when comparing different categories of contacts.

Fifth, contacts of cases identified towards the end of the study period had less time to develop symptoms and be tested than contacts identified earlier in the study period. The last case for which contact tracing data were collected was reported to PHA on 12 March 2020, meaning their contacts had at most two days to both develop symptoms and get tested. Although it was possible to identify five secondary cases that were hospitalised 


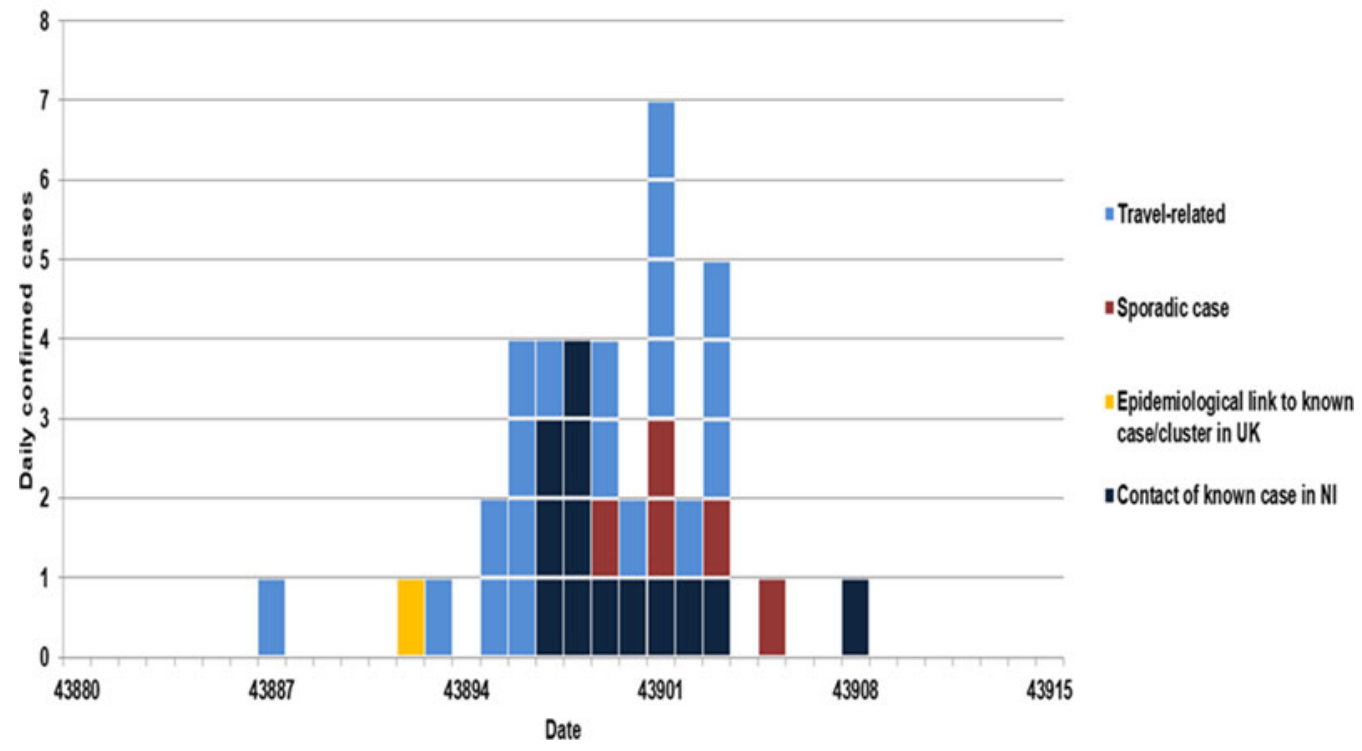

Fig. 4. COVID-19 cases identified through enhanced surveillance and contact tracing by specimen date and exposure, $\mathrm{NI}, 19 \mathrm{February} 2020$ to $25 \mathrm{March} 2020$ ( $n=39$ ).

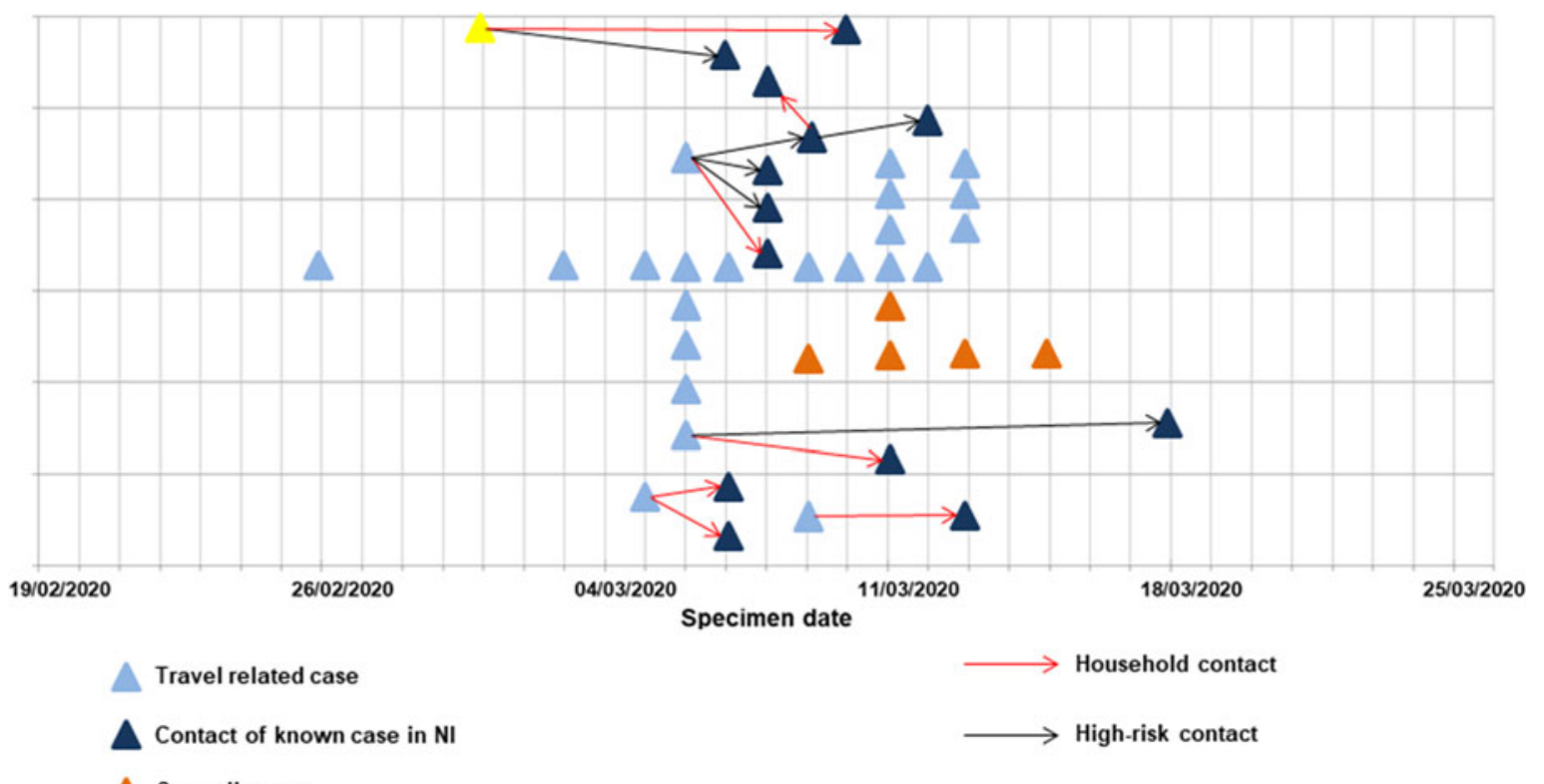

Sporadic case

Epidemiological link to known case/cluster in UK

Fig. 5. COVID-19 cases identified through enhanced surveillance and contact tracing, by specimen date, exposure, and transmission setting, NI, 19 February 2020 to 25 March $2020(n=39)$.

through linking contact tracing and virology data, any contacts that developed symptoms but did not require hospitalisation were not tested and therefore were not identified. This is again likely to result in underestimation of case numbers and attack rates in different categories of contacts, and may influence the estimates of the relative risk of infection when comparing different categories of contacts.

Sixth, it was not possible to determine how long cases were hospitalised for from the data available. Household contacts of hospitalised cases may potentially be at reduced risk of infection due to reduced exposure to the primary case following the primary case's hospitalisation, with the level of reduction of exposure dependent on the date and duration of hospitalisation. As we were not able to determine and adjust for this in our analysis, our estimate of the SAR among household contacts may be biased, with the assumption being that this bias may result in an underestimate of the SAR among household contacts.

\section{Discussion}

Cumulative incidence of COVID-19 in NI appears to be lower than the rest of the UK, and this may be due to one or more of 
Table 2. Exposures for COVID-19 cases identified through enhanced surveillance and contact tracing 26 February 2020 to 13 March 2020, $n=39$

\begin{tabular}{|c|c|c|}
\hline Exposures & $n$ & $\%$ \\
\hline \multicolumn{3}{|c|}{$\begin{array}{l}\text { Reported contact with individual(s) with COVID-19-like symptoms in } \\
\text { previous } 14 \text { days }\end{array}$} \\
\hline Yes & 19 & 48.7 \\
\hline No & 14 & 35.9 \\
\hline Unknown & 6 & 15.4 \\
\hline Travel to affected area outside UK & 20 & 51.3 \\
\hline Italy & 10 & 50.0 \\
\hline Austria & 7 & 35.0 \\
\hline France & 2 & 10.0 \\
\hline Cruise ship & 1 & 5.0 \\
\hline Contact of known case in $\mathrm{NI}$ & 13 & 33.3 \\
\hline Contact of known case/cluster in UK & 1 & 2.6 \\
\hline Sporadic case (no reported exposures) & 5 & 12.8 \\
\hline \multicolumn{3}{|l|}{ Occupation } \\
\hline $\mathrm{HCW}$ & 3 & 7.7 \\
\hline Pilot & 1 & 2.6 \\
\hline Retail manager & 1 & 2.6 \\
\hline Unknown & 34 & 87.2 \\
\hline
\end{tabular}

Table 3. Demographic characteristics of COVID-19 cases identified through enhanced surveillance and contact tracing, NI, 26 February 2020 to 18 March $2020, n=39$

\begin{tabular}{llc}
\hline Demographics & $n$ & $\%$ \\
\hline Male & 23 & 59.0 \\
\hline Age range & $13-86$ & \\
\hline Median age & 39 & \\
\hline Age group & & 0.0 \\
\hline$<5$ & 0 & 0.0 \\
\hline $5-10$ & 0 & 2.3 \\
\hline $10-20$ & 1 & 17.9 \\
\hline $20-30$ & 7 & 25.6 \\
\hline $30-40$ & 10 & 17.9 \\
\hline $40-50$ & 7 & 17.9 \\
\hline $50-60$ & 7 & 5.1 \\
\hline $60-70$ & 2 & 7.6 \\
\hline $70-80$ & 3 & 5.1 \\
\hline $80+$ & 2 & \\
\hline
\end{tabular}

several factors. NI's physical separation from the UK mainland may have resulted in relatively fewer importations and 'seeding' of SARS-CoV-2 cases before control measures were introduced. The first confirmed case in NI was also reported on 27 February 2020, nearly a month after the first case in England on 31 January, which may also explain the lower incidence in NI compared to the rest of the UK. The ROI, with which NI
Table 4. Clinical features of COVID-19 cases identified through enhanced surveillance and contact tracing, NI, 26 February 2020 to 18 March 2020, $n=39$

\begin{tabular}{ll}
\hline Clinical features & $N$ \\
\hline Symptoms $(n=36)$ & \\
\hline Fever & $25 / 36$ \\
\hline Cough & $24 / 36$ \\
\hline Shortness of breath & $8 / 36$ \\
\hline Chest pain & $3 / 36$ \\
\hline Headache & $3 / 36$ \\
\hline Fatigue & $2 / 36$ \\
\hline Coryzal & $2 / 36$ \\
\hline Myalgia & $1 / 36$ \\
\hline Delirium & $1 / 36$ \\
\hline No reported symptoms & $3 / 36$ \\
\hline Co-morbidities $(n=36)$ & $31 / 39$ \\
\hline Asthma & $2 / 39$ \\
\hline Hypertension & $1 / 36$ \\
\hline Tuberculosis & $2 / 36$ \\
\hline No reported co-morbidities & \\
\hline Admitted to hospital $(n=35)$ & \\
\hline Yes & \\
\hline Outcome $(n=39)$ & \\
\hline Died & \\
\hline Unknown & \\
\hline
\end{tabular}

shares an open border, policy of active case finding and testing throughout their epidemic may also have limited the number of potential cross-border importations of COVID-19. Phylogenetic analysis of early samples may enable better understanding of transmission lineages [19] and shed light on the role this may have played in the scale and progress of NI's COVID-19 epidemic. Given the different approaches to testing and surveillance between $\mathrm{NI}$ and the ROI, observed differences in cumulative incidence are difficult to interpret.

The data presented here also demonstrate the rapid transition to community transmission of SARS-CoV-2 following the first imported case in NI, and the mechanisms by which this transition may have taken place. It took just 11 days from the first laboratory-confirmed case in NI to the detection of the first sporadic case with no travel history or known links to confirmed cases. In our study we found that household contacts of individuals with laboratory-confirmed SARS-CoV-2 infection are at higher risk of both SARS-CoV-2 infection and symptomatic COVID-19 than high- and low-risk contacts. This finding adds to a growing body of evidence that households are an important locus of community transmission of SARS-CoV-2 [18-22]. Our estimate of the SAR among household contacts was $15.9 \%$ (95\% CI 6.6\%-30.1\%), which is similar to those reported in other studies, although higher SARs in household settings have been reported [25, 27]. This may be influenced by differences in testing policy, and/or the number and demographics of individuals occupying the same household, which may affect disease transmission and the susceptibility of individuals to infection. The average number of 
Table 5. Secondary and clinical attack rates among COVID-19 cases identified through enhanced surveillance and contact tracing, NI, 26 February 2020 to 18 March $2020, n=392$

\begin{tabular}{|c|c|c|c|c|c|}
\hline & Number of cases & Number of contacts & Proportion of contacts tested (\%) & Attack rate $(\%)$ & Clinical attack rate (\%) \\
\hline \multicolumn{6}{|l|}{ Risk category } \\
\hline Household & 7 & 44 & 15.9 & $15.9(6.6-30.1)$ & $15.9(6.6-30.1)$ \\
\hline High risk & 6 & 238 & 11.7 & $2.5(0.9-5.4)$ & $1.7(0.5-4.2)$ \\
\hline Low risk & 0 & 110 & 3.6 & 0.0 & 0.0 \\
\hline \multicolumn{6}{|c|}{ Age group $(n=259)^{a}$} \\
\hline$<5$ & 0 & 8 & 0.0 & 0.0 & 0.0 \\
\hline $5-10$ & 0 & 5 & 0.0 & 0.0 & 0.0 \\
\hline $10-20$ & 1 & 12 & 16.7 & $8.3(0.2-38.5)$ & $8.3(0.2-38.5)$ \\
\hline $20-30$ & 4 & 57 & 19.3 & $7.0(1.9-17.0)$ & $3.5(0.4-12.1)$ \\
\hline $30-40$ & 4 & 55 & 10.9 & $7.3(2.0-17.6)$ & $7.3(2.0-17.6)$ \\
\hline $40-50$ & 2 & 50 & 12.0 & $4.0(0.5-13.7)$ & $4.0(0.5-13.7)$ \\
\hline $50-60$ & 1 & 30 & 6.7 & $3.3(0.1-17.2)$ & $3.3(0.1-17.2)$ \\
\hline $60-70$ & 0 & 26 & 3.8 & 0.0 & 0.0 \\
\hline $70-80$ & 1 & 10 & 10.0 & $10.0(0.3-44.5)$ & $10.0(0.3-44.5)$ \\
\hline $80+$ & 0 & 5 & 0.0 & 0.0 & 0.0 \\
\hline
\end{tabular}

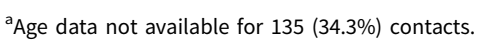

Table 6. Incubation periods and serial intervals for cases in six COVID-19 clusters identified in NI, 26 February 2020 to 18 March 2020, $n=13$

\begin{tabular}{|c|c|c|c|}
\hline & $\begin{array}{c}\text { Minimum } \\
\text { incubation } \\
\text { period (days) }\end{array}$ & $\begin{array}{c}\text { Maximum } \\
\text { incubation } \\
\text { period (days) }\end{array}$ & $\begin{array}{c}\text { Serial } \\
\text { interval } \\
\text { (days) }\end{array}$ \\
\hline \multicolumn{4}{|l|}{ Cluster A } \\
\hline Secondary case 1 & 1 & 5 & 5 \\
\hline Secondary case $2^{a}$ & NA & NA & NA \\
\hline Secondary case $3^{a}$ & NA & NA & NA \\
\hline Secondary case 4 & 3 & 4 & 6 \\
\hline \multicolumn{4}{|l|}{ Cluster B } \\
\hline Secondary case 1 & 1 & 7 & 7 \\
\hline Secondary case 2 & 1 & 6 & 6 \\
\hline \multicolumn{4}{|l|}{ Cluster C } \\
\hline Secondary case 1 & 1 & 5 & 2 \\
\hline Secondary case 2 & 3 & 3 & 2 \\
\hline \multicolumn{4}{|l|}{ Cluster D } \\
\hline Secondary case 1 & 9 & 12 & 9 \\
\hline Secondary case 2 & 5 & 5 & 3 \\
\hline \multicolumn{4}{|l|}{ Cluster E } \\
\hline Secondary case 1 & 1 & 6 & 3 \\
\hline Secondary case 2 & 12 & 16 & 13 \\
\hline \multicolumn{4}{|l|}{ Cluster F } \\
\hline Secondary case 1 & 2 & 9 & 8 \\
\hline
\end{tabular}

household contacts per primary case in our study was 1.6 (range: $0-5)$, which is lower than those reported in studies from China $[18,28]$ and South Korea [26]. Lower SAR in households with fewer than six members compared to householders with six or more members were also reported in a study from Guanzhou, China [23]. We did not find evidence of variation in the SAR by age group as reported by other studies $[6,18,23]$ and this is likely due to the small numbers in our study.

We found that, after adjusting for age and sex of contacts, the odds of testing positive for SARS-CoV-2 was 11.0 (95\% CI 1.770.3 ) times higher among household contacts of cases compared to non-household contacts, and this is within the range of estimates from a study in Hangzhou, China [27].

Despite the limitations of our study, this result combined with our estimate of the SAR among household contacts, suggests that control measures aimed at rapidly identifying, testing and isolating household contacts of cases regardless of whether or not they are symptomatic, and reducing the risk of infection in the household, may be effective in preventing transmission of SARS-CoV-2. Rapid identification and testing of household contacts takes on added importance given that we found evidence of possible presymptomatic transmission in our study.

As it will still be some time before a vaccine or vaccines are widely available, further research into the effectiveness of nonpharmaceutical interventions (NPIs) in preventing SARS-CoV-2 transmission in the UK is urgently needed to strengthen the public health response to the pandemic, and identify effective interventions to reduce SARS-CoV-2 transmission in the household. One such intervention that may be effective is the use of face coverings and masks. Several recent studies have generated evidence on the effectiveness of face coverings and masks at reducing the risk of SARS-CoV-2 infection generally [27, 29-33], and at reducing transmission within households. Results from a study in China [28] on face mask use among household contacts of 
Table 7. Logistic regression analysis of association between contact risk category, age and sex and testing positive for SARS-CoV-2, $n=44$

\begin{tabular}{|c|c|c|c|c|}
\hline & Crude odds ratio $(95 \% \mathrm{Cl})$ & $P$-value & Adjusted odds ratio $(95 \% \mathrm{Cl})$ & $P$-value \\
\hline High- and low-risk contacts & 1.0 & & & \\
\hline Household & $16.9(2.8-102.4)$ & 0.002 & $11.0(1.7-70.3)$ & 0.011 \\
\hline Age & $1.0(0.9-1.1)$ & 0.770 & $1.0(0.9-1.1)$ & 0.884 \\
\hline Male & $0.4(0.1-1.5)$ & 0.185 & $0.5(0.1-2.9)$ & 0.462 \\
\hline
\end{tabular}

Note: The significance of bold is to highlight that only being a household contact showed statistical evidence of being associated with higher odds of testing positive.

individuals with laboratory-confirmed SARS-CoV-2 infection suggest that one or more household members (including the primary case) wearing a face mask at home prior to symptom onset in the primary case was $79 \%$ (95\% CI $21 \%-94 \%)$ effective at reducing transmission in the home, although it should be noted that data on mask use by study participants was collected via selfreporting rather than direct observation by the study authors. As the authors of this study highlight, the use of face masks have been shown to be effective in reducing transmission of other respiratory viruses [21], including within households under randomised-control trial conditions [34]. Another study from China [25] reported that households that reported not adopting protective measures (defined as wearing masks when contact with index case, hand hygiene after contact with index case and avoiding contact with the index case) after illness onset in the index case had SARs 4.95 (95\% CI 1.59-15.39) times higher than households that did report adopting these measures.

Other interventions to prevent transmission within households should also be investigated, and the household setting may present additional opportunities for outbreak control that are not feasible in other areas with increased risk of SARS-CoV-2 transmission such as healthcare facilities, care homes and other institutional settings, especially during periods of increased transmission. A study on household transmission in China reported no secondary SARS-CoV-2 infections among household contacts of cases who self-quarantined at home at the time of symptom onset [18]. Another study from China also reported associations between reduced contact between household members and lower risk of secondary SARS-CoV-2 infection within the household [28]. The ability of individuals to self-quarantine effectively at home is only possible, however, if the household is uncrowded and has sufficient space for infected individuals to minimise contact with other household residents [25, 28]. Future research on reducing household transmission of SARS-CoV-2 could take this further and investigate the effectiveness, feasibility and acceptability of quarantining individuals outside of the home if their household conditions are assessed to be unsuitable for self-quarantine.

Identifying and testing potential interventions takes on added importance given anticipated increased levels of COVID-19 and seasonal increases in other diseases during the coming winter. Preliminary research suggests compliance with 'lockdown' measures in the UK has been high [35] despite the many and varied challenges these restrictions on everyday life have imposed, so there may be cause to be optimistic that uptake of interventions aimed at reducing transmission within households may be high if they are tailored to a range of audiences, communicated clearly and implemented effectively [36].

Supplementary material. The supplementary material for this article can be found at https://doi.org/10.1017/S0950268821000224

Data availability statement. The cumulative incidence and mortality data that support the findings of this study are openly available through the COVID-19 data dashboards published by the Northern Ireland Department of Health, Public Health England, Public Health Wales, The Government of Scotland and The Government of Ireland. The enhanced surveillance data and contact tracing data used in the study cannot be made available due to data protection requirements.

\section{References}

1. WHO. Disease outbreak news - update - novel coronavirus - China Geneva, Switzerland 2020 (12 January 2020). Available at https://www. who.int/csr/don/12-january-2020-novel-coronavirus-china/en/.

2. Wang D et al. (2020) Clinical characteristics of 138 hospitalized patients with 2019 novel coronavirus-infected pneumonia in Wuhan, China. Journal of the American Medical 323, 1061-1069.

3. Deng Y et al. (2020) Clinical characteristics of fatal and recovered cases of coronavirus disease 2019 in Wuhan, China: a retrospective study. China Medical Journal (English) 133, 1261-1267.

4. Huang C et al. (2020) Clinical features of patients infected with 2019 novel coronavirus in Wuhan, China. Lancet (London, England) 395, 497-506.

5. Zheng F et al. (2020) Clinical characteristics of children with coronavirus disease 2019 in Hubei, China. Current Medical Science 40, 275-280.

6. Chen P et al. (2020) Clinical and demographic characteristics of cluster cases and sporadic cases of coronavirus disease 2019 (COVID-19) in 141 patients in the main district of Chongqing, China, between January and February 2020. Medical Science Monitor. 26, e923985.

7. Zhao XY et al. (2020) Clinical characteristics of patients with 2019 coronavirus disease in a non-Wuhan area of Hubei Province, China: a retrospective study. Biomed Central Infectious Diseases 20, 311.

8. Qiu C et al. (2020) Transmission and clinical characteristics of coronavirus disease 2019 in 104 outside-Wuhan patients, China. Journal of Medical Virology 92, 2027-2035.

9. Guan WJ et al. (2020) Clinical characteristics of coronavirus disease 2019 in China. New England Journal of Medicine 382, 1708-1720.

10. Zhang J et al. (2020) Evolving epidemiology and transmission dynamics of coronavirus disease 2019 outside Hubei province, China: a descriptive and modelling study. The Lancet Infectious Diseases 20, 793-802.

11. Department of Health Northern Ireland. The public health notifiable diseases order (Northern Ireland) 2020 Belfast, Northern Ireland 2020. Available at https://www.legislation.gov.uk/nisr/2020/23/made.

12. PHE. Laboratory reporting to Public Health England: a guide for diagnostic laboratories London, UK: PHE; 2016. Available at https://assets. 
publishing.service.gov.uk/government/uploads/system/uploads/attachment_data/file/739854/PHE_Laboratory_Reporting_Guidelines.pdf.

13. WHO (2020) The First Few X (FFX) cases and contact investigation protocol for 2019-novel coronavirus (2019-nCoV) infection. Geneva, Switzerland: WHO; 10 February 2020.

14. Coronavirus (COVID-19) in the UK: Government of the UK. Available at https://coronavirus.data.gov.uk/.

15. Public Health Wales - Rapid COVID-19 Surveillance: Public Health Wales. Available at https://public.tableau.com/profile/public.health.wales. health.protection\#!/vizhome/RapidCOVID-19virology-Public/ Headlinesummary.

16. Coronavirus (COVID-19): daily data for Scotland: Government of Scotland. Available at https://www.gov.scot/publications/coronaviruscovid-19-daily-data-for-scotland/.

17. Ireland's COVID-19 Data Hub: Government of Ireland. Available at https://covid19ireland-geohive.hub.arcgis.com/.

18. Li W et al. (2020) The characteristics of household transmission of COVID-19. Clinical Infectious Diseases 71, 1943-1946.

19. Oliver Pybus AR et al. on behalf of the COG-UK consortium. (2020) Preliminary analysis of SARS-CoV-2 importation \& establishment of UK transmission lineages. Report. London, UK: COG-UK Consortium; 08 June 2020.

20. Bi Q et al. (2020) Epidemiology and transmission of COVID-19 in 391 cases and 1286 of their close contacts in Shenzhen, China: a retrospective cohort study. The Lancet Infectious Diseases 20, 911-919.

21. Lo JY et al. (2005) Respiratory infections during SARS outbreak, Hong Kong, 2003. Emerging Infectious Diseases 11, 1738-1741.

22. Burke RM et al. (2020) Active monitoring of persons exposed to patients with confirmed COVID-19 - United States, January-February 2020. Morbidity and Mortality Weekly Report 69, 245-246.

23. Jing Q-L et al. (2020) Household secondary attack rate of COVID-19 and associated determinants in Guangzhou, China: a retrospective cohort study. The Lancet Infectious Diseases 20, 1141-1150.

24. Ge Y and Sun S (2020) Estimation of coronavirus disease case-fatality risk in real time. Emerging Infectious Diseases 26, 1922-1923.
25. Wu J et al. (2020) Household transmission of SARS-CoV-2, Zhuhai, China, 2020. Clinical Infectious Diseases 71, 2099-2108.

26. Covid-19 National Emergency Response Center E, Case Management Team KCfDC, Prevention (2020) Coronavirus disease-19: summary of 2370 contact investigations of the first 30 cases in the Republic of Korea. Osong Public Health Research and Perspectives 11, 81-84.

27. Wu Y et al. (2020) Risk of SARS-CoV-2 infection among contacts of individuals with COVID-19 in Hangzhou, China. Public Health 185, 57-59.

28. Wang Y et al. (2020) Reduction of secondary transmission of SARS-CoV-2 in households by face mask use, disinfection and social distancing: a cohort study in Beijing, China. British Medical Journal Global Health 5, e002794.

29. Leung NHL et al. (2020) Respiratory virus shedding in exhaled breath and efficacy of face masks. Nature Medicine 26, 676-680.

30. Lyu W and Wehby GL (2020) Community use of face masks and COVID-19: evidence from a natural experiment of state mandates in the US. Health Affairs(Millwood 39, 1419-1425.

31. Zhang R et al. (2020) Identifying airborne transmission as the dominant route for the spread of COVID-19. Proceedings of the National Academy of Sciences of the United States of America 117, 14857-14863.

32. Payne DC et al. (2020) SARS-CoV-2 infections and serologic responses from a sample of U.S. navy service members - USS Theodore Roosevelt, April 2020. Morbidity and Mortality Weekly Report 69, 714-721.

33. Hendrix MJ et al. (2020) Absence of apparent transmission of SARS-CoV-2 from two stylists after exposure at a hair salon with a universal face covering policy - Springfield, Missouri, May 2020. Morbidity and Mortality Weekly Report 69, 930-932.

34. MacIntyre CR et al. (2009) Face mask use and control of respiratory virus transmission in households. Emerging Infectious Diseases 15, 233-241.

35. Benjamin Jeffrey CEW and Ainslie KEC et al. (2020) Anonymised and aggregated crowd level mobility data from mobile phones suggests that initial compliance with COVID-19 social distancing interventions was high and geographically consistent across the UK. London, UK: Imperial College London; 29 May 2020.

36. Javid B, Weekes MP and Matheson NJ (2020) Covid-19: should the public wear face masks? British Medical Journal 369, m1442. 